\title{
The human Periapical Cyst- Mesenchymal Stem Cells (hPCy-MSCs): an useful newly discovered source for MSCs to use in regenerative medicine
}

\section{Background}

The regenerative medicine has passed through several different phases: after the enthusiasm of the early stages, in which all the researchers were experimenting the methodologies for regeneration, although "demolitive", today, we search highly conservative tissue regeneration, and, as much as possible physiological.

The searching for alternative biological sites, easily accessible, free of morbidity for the patient, rich in stem cells, is the current challenge of regenerative medicine. Human periapical inflammatory tissues that are formed resulting from endodontic infection are usually termed periapical granuloma, and the condition is referred to as apical periodontitis. This pathological condition, if untreated, can lead to a periapical cyst.

\section{The research}

The aim of the research team of "Tecnologica Research Institute" from Italy was to determine if cells isolated from human periapical inflammatory cyst can express mesenchymal stem-like properties.

The results of these studies were published a few months ago in the prestigious international journal "International Journal of Biological Sciences", inside the scientific article entitled "Cells Isolated from Human Periapical Cysts Express Mesenchymal Stem Cell-like Properties", written by Marrelli et al.'

In this scientific publication have been disclosed to the scientific community the results of a study that showed that the dental periapical cysts contain in their wall a multitude of cell types, including mesenchymal stem cells. This is the first time in the world, which is documented in a scientific way this data, in fact, the team of Tecnologica Research Institute has coined a term that identifies the new cell line: the hPCy-MSCs (human periapical cyst-Mesenchymal Stem Cells).

The dental periapical inflammatory cysts, also known as apicalradicular cysts, are those space-occupying formations that usually develop as a result of pulpal necrosis, the biological "death" of the tooth, and that are often discovered by the dentist during the observation of a common orthopanoramic radiography; dental periapical cysts are among the most common dental radiographic findings, because of the ease of their development, but they are not usually dangerous from a histopathological point of view, however, they are commonly identified as a clinical finding to be eradicated as soon as possible, often together with the necrotic tooth that has made them develop.

Human periapical cystic tissue analyzed in the study was obtained from healthy volunteers donors. The cysts received several washes in sterile phosphatase buffer saline (PBS, Invitrogen) containing antibiotics. Cells from human periapical cystic tissue were isolated and characterized by flow cytofluorimetry: these cells expressed mesenchymal stem-like properties, so the authors termed them "human Periapical Cyst- Mesenchymal Stem Cells" (hPCy-MSCs).

\author{
Volume I Issue I - 2014 \\ Marco Tatullo
Tecnologica Research Institute, Italy
}

Correspondence: Marco Tatullo, Scientific DirectorTecnologica Research Institute, St. E. Fermi - Crotone, Italy, Tel 0039-0962-930362, Email marco.tatullo@tecnologicasrl.com

Received: April 24, 2014 | Published: April 25, 2014

No study has up to now evaluated the possible existence of MSCs in human periapical cysts. In this research, the authors have demonstrated that cells isolated from human periapical inflammatory cysts display MSC-like properties, as evidenced by the expression of the MSC markers $\mathrm{CD} 13^{+}, \mathrm{CD} 29^{+}, \mathrm{CD} 44^{+}, \mathrm{CD} 73^{+}, \mathrm{CD} 90^{+}, \mathrm{CD} 105^{+}$, $\mathrm{CD} 45^{-}, \mathrm{STRO}^{-} 1^{+}, \mathrm{CD}_{146^{+}}$as well as their self-renewal capability and osteogenic and adipogenic potential. Thus, it's actually possible to successfully isolated and characterised hPCy-MSCs from human periapical cysts, without recourse to the removal of biologically healthy tissues, since human periapical cysts are destined for surgical removal to prevent the development of disabling, pathological conditions.

\section{Conclusion}

Up to now, the most common sources of adult stem cells were usually identified in bone marrow, adipose tissue, and, recently, in some structures such as intraoral dental pulp. The dental inflammatory periapical cysts are usually not dangerous from a histopathological point of view, on the contrary, they represent a neoformation absolutely free of utility for the patient, in fact, usually the cysts are stored in biological waste: the possibility of obtaining mesenchymal stem cells from a "biological waste" puts us in a position to really take advantage of an "alternative" source, at practically zero biological cost. The team of Tecnologica Research Institute has discovered, isolated and characterized cells from inflamed periapical cyst, which have the characteristics of mesenchymal stem cells. Therefore, I am sure that hPCy-MSCs could have many potential regenerative properties if we'll use them in the future medical applications.

\section{Acknowledgments}

None.

\section{Conflict of interest}

The author declares that there is no conflict of interest.

\section{References}

1. Marrelli M, Paduano F, Tatullo M. Cells isolated from human periapical cysts express mesenchymal stem cell-like properties. Int $\mathrm{J}$ Biol Sci. 2013;9(10):1070-1078. 